University of Nebraska - Lincoln

DigitalCommons@University of Nebraska - Lincoln

Faculty Publications from the Department of Electrical \& Computer Engineering, Department Electrical and Computer Engineering

2012

\title{
Compensation Algorithms for Sliding Mode Observers in Sensorless Control of IPMSMs
}

\author{
Yue Zhao \\ University of Nebraska-Lincoln, yue.zhao@huskers.unl.edu \\ Wei Qiao \\ University of Nebraska-Lincoln, wqiao@engr.unl.edu \\ Long Wu \\ Phoenix International - A John Deere Company, WuLong@JohnDeere.com
}

Follow this and additional works at: https://digitalcommons.unl.edu/electricalengineeringfacpub

Part of the Electrical and Computer Engineering Commons

Zhao, Yue; Qiao, Wei; and Wu, Long, "Compensation Algorithms for Sliding Mode Observers in Sensorless Control of IPMSMs" (2012). Faculty Publications from the Department of Electrical and Computer Engineering. 200.

https://digitalcommons.unl.edu/electricalengineeringfacpub/200

This Article is brought to you for free and open access by the Electrical \& Computer Engineering, Department of at DigitalCommons@University of Nebraska - Lincoln. It has been accepted for inclusion in Faculty Publications from the Department of Electrical and Computer Engineering by an authorized administrator of DigitalCommons@University of Nebraska - Lincoln. 


\section{Compensation Algorithms for Sliding Mode Observers in Sensorless Control of IPMSMs}

\author{
Yue Zhao, Wei Qiao \\ Department of Electrical Engineering \\ University of Nebraska-Lincoln \\ Lincoln, NE 68588-0511 USA \\ yue.zhao@huskers.unl.edu; wqiao@engr.unl.edu
}

\author{
Long $\mathrm{Wu}$ \\ Phoenix International - A John Deere Company \\ 1750 NDSU Research Park Dr. \\ Fargo, ND 58102 \\ WuLong@JohnDeere.com
}

\begin{abstract}
Electromechanical sensors are commonly used to obtain rotor position/speed for high-performance control of interior permanent magnet synchronous machines (IPMSMs) in vehicle systems. However, the use of these sensors increases the cost, size, weight, wiring complexity and reduces the mechanical robustness of IPMSM drive systems. These issues, together with some practical requirements, e.g., wide speed range, extreme environment temperature, and adverse loading conditions, make a sensorless control scheme desirable. This paper proposes an extended back electromotive force (EMF)-based sliding mode rotor position observer for sensorless vector control of IPMSMs. Based on filter characteristics, a robust compensation algorithm is developed to improve the performance of the sliding-mode observer (SMO). Multistage-filter and dual-filter schemes are designed to further improve the steady-state and transient performance, respectively, of the compensation algorithms. The proposed SMO and compensation algorithms are validated by simulations in MATLAB Simulink as well as experiments on a practical IPMSM drive system.
\end{abstract}

Keywords-compensation; filter design; interior permanent magnet synchrnous machine (IPMSM); sensorless control; sliding mode observer (SMO)

\section{INTRODUCTION}

IPMSMs are widely used in electric vehicle systems due to their advantages, such as high efficiency and high power density. Accurate information of rotor position is indispensable for high-performance control of IPMSMs. Electromechanical sensors, e.g., resolvers, optical encoders, and hall-effect sensors, are commonly used to obtain rotor position/speed in IPMSM drives. The use of these sensors increases cost, size, weight, and wiring complexity of IPMSM drive systems. From the viewpoint of system reliability, mounting electromechanical sensors on rotors will reduce mechanical robustness of electric machines. The noise in harness and harness break will be fatal to the control system. Moreover, sensors are subjected to high failure rates in harsh environments, such a high environment temperature, highspeed operation, and adverse or heavy load conditions [1]. To overcome these drawbacks, much research effort has gone into the development of sensorless drives that have comparable or similar dynamic performance to the sensor-based drives during the last decades.
There are two major approaches in the literature for rotor position/speed observation in sensorless control of IPMSMs. One is based on the extraction of the position information from the estimated back EMF [2]-[4] and the other is based on the rotor saliency, e.g., signal injection method [5]. In the back EMF-based methods, disturbance observers [2], D-state observer [6], and SMOs [3], [7] have been developed to estimate the rotor position from the estimated back EMF. The back EMF-based methods can perform well in the mediumand high-speed regions. However, since the value of the back EMF is too small to be estimated accurately in the standstill and low-speed region, the back EMF-based methods usually do not work well in these conditions. Signal injections are commonly used to help with rotor position observation for sensorless control in low-speed regions. Therefore, in order to ensure acceptable performance of the sensorless control over the entire operating range, multiple methods may need to be combined for rotor position observation.

Among different observers, the SMO is a promising one. In several previous works, the SMO has been applied to the surface mounted PMSM drives [8]-[10]. The block diagram of a typical back EMF-based SMO for rotor position estimation is shown in Fig. 1. The SMO uses a discontinuous control (i.e., the switching block) to estimate the back EMF based on the errors of the stator current estimation. There are several options to design the switching block, including sign functions, saturation functions, and sigmoid functions. The low-pass filter is used to extract the back EMF $\hat{e}_{\alpha \beta}$ from the output $Z_{\alpha \beta}$ of the switching block. Since the fundamental frequency of $Z_{\alpha \beta}$ varies with the rotor speed of the PMSM, the low-pass filter with a constant cutoff frequency will have variable delays (i.e., phase shift) at different rotor speeds. Therefore, appropriate compensation algorithms are needed to compensate for this varying phase shift of the low-pass filter in order to accurately estimate the rotor position of the PMSM.

This paper proposes an extended back EMF-based SMO for rotor position and speed observation in the sensorless IPMSM drives. Robust compensation algorithms are developed to ensure accurate observation of the rotor position over a wide speed range of the IMPSM. The proposed compensation algorithms, rotor position observer, and sensorless control are validated by simulations in MATLAB Simulink as well as experiments for an IPMSM drive system. 


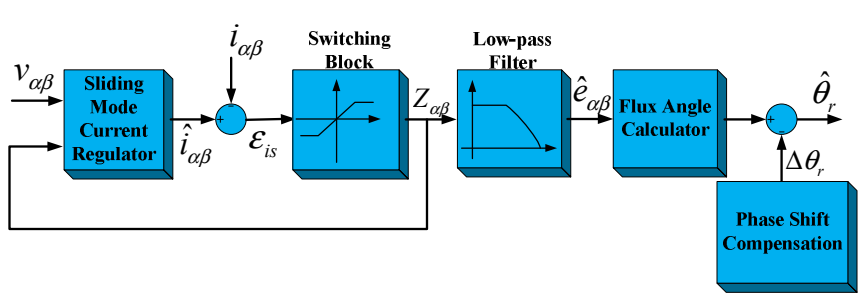

Fig. 1. Block diagram of a back EMF-based sliding mode rotor position observer.

\section{DYNAMIC MODEL OF AN IPMSM}

The dynamics of an IPMSM can be modeled in the $d-q$ rotating reference frame as:

$$
\left[\begin{array}{l}
v_{d} \\
v_{q}
\end{array}\right]=\left[\begin{array}{cc}
R+p L_{d} & -\omega_{r e} L_{q} \\
\omega_{r e} L_{d} & R+p L_{q}
\end{array}\right]\left[\begin{array}{c}
i_{d} \\
i_{q}
\end{array}\right]+\left[\begin{array}{c}
0 \\
\omega_{r e} \psi_{m}
\end{array}\right]
$$

where $p$ is the derivative operator; $v_{d}, v_{q}, i_{d}$, and $i_{q}$ are the stator voltages and currents, respectively; $\omega_{r e}$ is the rotor electrical angular speed; $L_{d}$ and $L_{q}$ are the $d$-axis and $q$-axis inductances, respectively; and $R$ is the stator resistance. Using inverse Park transformation the dynamic model of the IPMSM in the $\alpha-\beta$ stationary reference frame can be expressed as:

$$
\begin{aligned}
{\left[\begin{array}{c}
v_{\alpha} \\
v_{\beta}
\end{array}\right]=} & p\left[\begin{array}{cc}
L+\Delta L \cos \left(2 \theta_{r e}\right) & \Delta L \sin \theta_{r e} \\
\Delta L \sin \theta_{r e} & L-\Delta L \cos \left(2 \theta_{r e}\right)
\end{array}\right]\left[\begin{array}{c}
i_{\alpha} \\
i_{\beta}
\end{array}\right] \\
& +R\left[\begin{array}{c}
i_{\alpha} \\
i_{\beta}
\end{array}\right]+\omega_{r e} \psi_{m}\left[\begin{array}{c}
-\sin \theta_{r e} \\
\cos \theta_{r e}
\end{array}\right]
\end{aligned}
$$

where $L=\frac{L_{d}+L_{q}}{2} ; \Delta L=\frac{L_{d}-L_{q}}{2} ;$ and $\theta_{r e}$ is the rotor position angle.

Due to the saliency of the IPMSM (i.e., $L_{d} \neq L_{q}$ ), both the back EMF and the inductance matrix contain the information of the rotor position angle. Moreover, (2) contains both $2 \theta_{r e}$ and $\theta_{r e}$ terms, which is not easy for mathematical processing to obtain the rotor position from the back EMF directly. To facilitate the rotor position observation, an extended back EMF-based model for IPMSMs is proposed in [2] as follows:

$$
\begin{aligned}
& {\left[\begin{array}{c}
v_{\alpha} \\
v_{\beta}
\end{array}\right]=\left[\begin{array}{cc}
R+p L_{d} & \omega_{r e}\left(L_{d}-L_{q}\right) \\
\omega_{r e}\left(L_{q}-L_{d}\right) & R+p L_{d}
\end{array}\right]\left[\begin{array}{l}
i_{\alpha} \\
i_{\beta}
\end{array}\right]} \\
& +\underbrace{\left[\left(L_{d}-L_{q}\right)\left(\omega_{r e} i_{d}-p i_{q}\right)+\omega_{r e} \psi_{m}\right]\left[\begin{array}{c}
-\sin \theta_{r e} \\
\cos \theta_{r e}
\end{array}\right]}_{\text {extended back EMF }}
\end{aligned}
$$

In (3) only the extended back EMF term contains the information of the rotor position. If the extended back EMF can be estimated, the rotor position can be obtained directly.

\section{Design of the Sliding Mode Rotor Positton OBSERVER}

Generally speaking, a SMO is an observer whose input is a discontinuous function of the error between the estimated and measured system states [11]. For example, the discontinuous function (i.e., the switching block) of the sliding mode rotor position observer in Fig. 1 uses the stator current estimation errors as the input. If the sliding mode manifold is well designed and when the estimated currents reach the manifold, the sliding mode will be enforced. The system's dynamic behavior in the sliding mode only depends on the surfaces chosen in the state space and is not affected by the system structure and parameter uncertainty. Therefore, the SMO has some inherit advantages, including order reduction, disturbance rejection, and strong robustness.

Let $k$ denote the $\left(L_{d}-L_{q}\right)\left(\omega_{r e} i_{d}-p i_{q}\right)+\omega_{r e} \psi_{m}$ term, the IPMSM current model (3) can be written as:

$$
\left\{\begin{array}{l}
\frac{d i_{\alpha}}{d t}=\frac{v_{\alpha}}{L_{d}}-\frac{R}{L_{d}} i_{\alpha}+\omega_{r e} \frac{L_{q}-L_{d}}{L_{d}} i_{\beta}+\frac{k}{L_{d}} \sin \theta_{r e} \\
\frac{d i_{\beta}}{d t}=\frac{v_{\beta}}{L_{d}}-\frac{R}{L_{d}} i_{\beta}-\omega_{r e} \frac{L_{q}-L_{d}}{L_{d}} i_{\alpha}-\frac{k}{L_{d}} \cos \theta_{r e}
\end{array}\right.
$$

A sliding mode current observer is designed with the same structure as the IPMSM model:

$$
\left\{\begin{array}{l}
\frac{d \hat{i}_{\alpha}}{d t}=\frac{v_{\alpha}^{*}}{L_{d}}-\frac{R}{L_{d}} \hat{i}_{\alpha}+\omega_{r e} \frac{L_{q}-L_{d}}{L_{d}} i_{\beta}+\frac{k}{L_{d}} l \times Z_{\alpha} \\
\frac{d \hat{i}_{\beta}}{d t}=\frac{v_{\beta}^{*}}{L_{d}}-\frac{R}{L_{d}} \hat{i}_{\beta}-\omega_{r e} \frac{L_{q}-L_{d}}{L_{d}} i_{\alpha}-\frac{k}{L_{d}} l \times Z_{\beta}
\end{array}\right.
$$

$\operatorname{In}_{*}(5), l$ is the feedback gain of the switching control vector $Z_{\alpha \beta} ; v_{\alpha}^{*}$ and $v_{\beta}^{*}$ are the commanded voltages obtained from the current regulated vector control of the IPMSM. If the deadtime effect of the inverter is ignored or well compensated, $v_{\alpha \beta}{ }_{\alpha \beta}$ should be identical to the $v_{\alpha \beta}$ measured from the IPMSM stator terminals. Subtracting (5) from (4) the following equations can be obtained.

$$
\left\{\begin{array}{l}
\frac{d\left(i_{\alpha}-\hat{i}_{\alpha}\right)}{d t}=-\frac{R}{L_{d}}\left(i_{\alpha}-\hat{i}_{\alpha}\right)+\frac{k}{L_{d}} \sin \theta_{r e}-\frac{k l}{L_{d}} Z_{\alpha} \\
\frac{d\left(i_{\beta}-\hat{i}_{\beta}\right)}{d t}=-\frac{R}{L_{d}}\left(i_{\alpha}-\hat{i}_{\alpha}\right)-\frac{k}{L_{d}} \cos \theta_{r e}+\frac{k l}{L_{d}} Z_{\beta}
\end{array}\right.
$$

Let $e_{\alpha \beta}^{T}=\left[\begin{array}{ll}k / L_{d} \sin \theta_{r e} & -k / L_{d} \cos \theta_{r e}\end{array}\right], Z_{\alpha \beta}^{T}=\left[\begin{array}{ll}Z_{\alpha} & -Z_{\beta}\end{array}\right]$, and $S^{T}=\left[\begin{array}{ll}i_{\alpha}-\hat{i}_{\alpha} & i_{\beta}-\hat{i}_{\beta}\end{array}\right],(6)$ can be expressed as:

$$
\dot{S}^{T}=-\frac{R}{L_{d}} S^{T}+e_{\alpha \beta}^{T}-\frac{k l}{L_{d}} Z_{\alpha \beta}^{T}
$$

Since the extended back EMF are bounded, they can be suppressed by the discontinuous input with $l>\max \left\{\left|e_{\alpha}\right|,\left|e_{\beta}\right|\right\}$. When the system is enforced to the sliding mode, $\dot{S}^{T}=S^{T}=0$ and $e_{\alpha \beta}^{T}=k l / L_{d} \cdot Z_{\alpha \beta}^{T}$. Furthermore, the rotor position angle $\theta_{r e}$ can be estimated as:

$$
\hat{\theta}_{r e}=-\tan ^{-1}\left(\frac{e_{\alpha}}{e_{\beta}}\right)=\tan ^{-1}\left(\frac{Z_{\alpha}}{Z_{\beta}}\right)
$$

\section{OVERALl IPMSM SENSORLESS CONTROL SYSTEM}

The IPMSM drives in electric vehicle applications require to be operated in a wide speed range. In the high-speed range, or the flux-weakening operation, the optimal stator current commands are not only functions of commanded torque and speed but also functions of IPMSM parameters and the DC bus voltage of the inverter [12]. In order to have a stable and fast response, a feedforward controller with several lookup tables is used in this work. 
Fig. 2 shows the overall block diagram of a sensorless control system for IPMSMs. The control system consists of a speed PI regulator, which is used to generate the command torque based on the speed error. The base torque is the maxium torque at each speed point, and this can be obtained from a 2-D lookup table. As mentioned before DC link voltage will also effect the current command, so a speed-voltage ratio is also used. The dq current commands are genertated from two lookup tables based on torque percentage and speed-voltage ratio. In addition, current PI regulator with feedforward voltage compensation, and convertional modules for vector control such as space-vector pulse-width modulation (SVPWM) gernerator, 3-phase inverter, Park transformaton are also included.

In this sensorless controller, the rotor position is obtained by the sliding mode position observer, and the speed is calculated by a circular buffer based on the estimated rotor position. In this position buffer, the change in the position between two consecutive steps and the corresponding time duration are written into the buffer. The buffer size can be ajusted according to the accuracy requirment for the speed and the transient response.

There are two operation modes for this controller. One is an open-loop control mode, in which the controller uses the measured rotor position and speed; and the SMO uses the command voltages $v_{\alpha}{ }_{\alpha}$ and $v_{\beta}{ }_{\beta}$ as well as the measured currents $i_{a}$ and $i_{b}$ to estimate the rotor position. The estimated rotor position is then compared with the measured rotor position to evaluate the performance of the SMO. The other mode is a close-loop control mode, in which the estimated rotor position and speed are feedback into the controller. In the low-speed region, the magnitude of the back EMF is too small to be estimate accurately. Therefore, a starting algorithm is designed to accelerate the motor to a minimum transition speed, and then enable the SMO for the close-loop sensorless control.

\section{COMPENSATION ALGORITHMS}

\section{A. Simulation Results and Problem Description}

The proposed SMO is integrated into the vector control of an IPMSM. The parameters of the IPMSM are as follows: the rated power is $155 \mathrm{~kW}$; the base speed is $5,000 \mathrm{RPM}$; and the stator phase resistance $R_{s}=0.01 \Omega$. Since the machine inductances vary with the stator currents, their values are stored in a lookup table and the averaging values for $L_{d}$ and $L_{q}$ are $0.2 \mathrm{mH}$ and $0.79 \mathrm{mH}$, respectively. The DC bus voltage of the inverter is $700 \mathrm{~V}$. The frequency of the SVPWM is $6 \mathrm{kHz}$. The system is simulated in MATLAB Simulink.

The simulation results for the open-loop system are shown in Fig. 3, including a comparison of the commanded and actual rotor speeds, the error between the commanded and actual rotor speeds, and the error between the actual and estimated rotor position without any compensation, where the open-loop system means that the estimated rotor position has not been used as an input to the control system. As shown in Fig. 3, the commanded speed accelerates from 0 to 5,000 RPM at a rate of 2,500 RPM/s and reaches the steady-state speed of $5,000 \mathrm{RPM}$ at $2 \mathrm{~s}$. The results show that the position error is not a constant value but varies with the rotor speed because the phase delay of the SMO depends on the rotor speed. The load torque is shown in Fig. 4, which maintains a constant value of $50 \mathrm{Nm}$ during acceleration and varies after $2.1 \mathrm{~s}$.

\section{B. Compensation Algorithms}

Algorithms for compensating filter delays have been discussed in several previous works. As shown in Fig. 3, the position error curve can be approximated by a linear function or a higher order polynomial [9]. However, this compensation method is based on the measured error curve and not robust to speed variations.

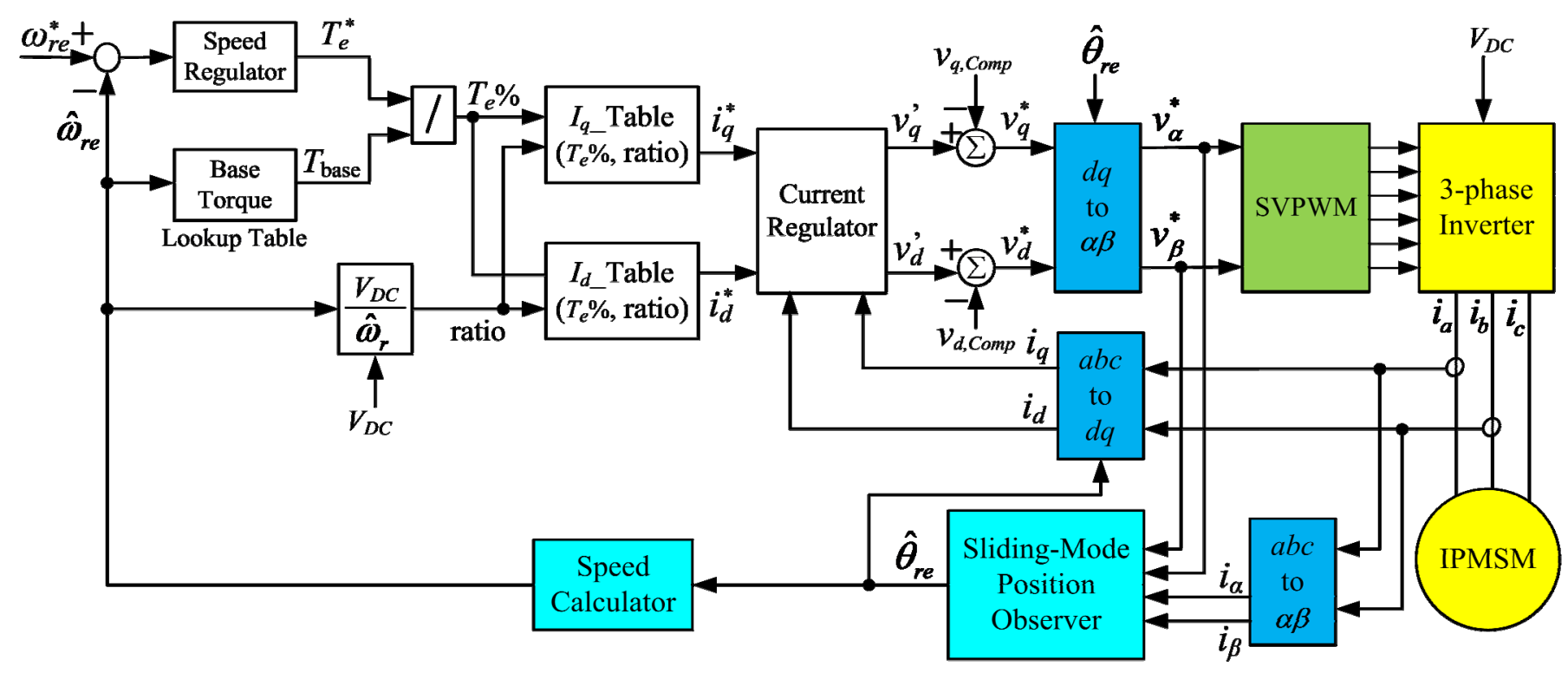

Fig. 2. Block diagram of the proposed sensorless control scheme. 

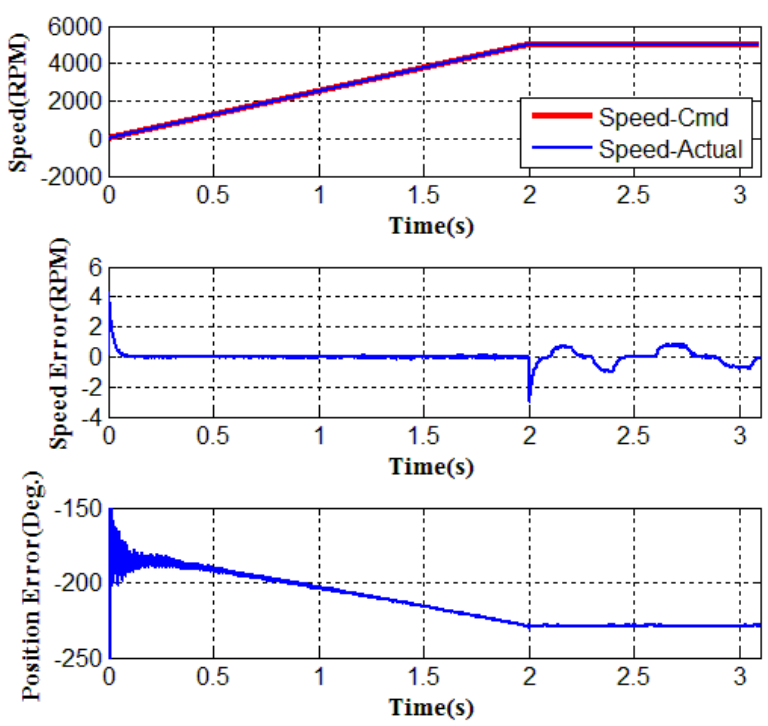

Fig. 3. Simulation results for the open-loop system.

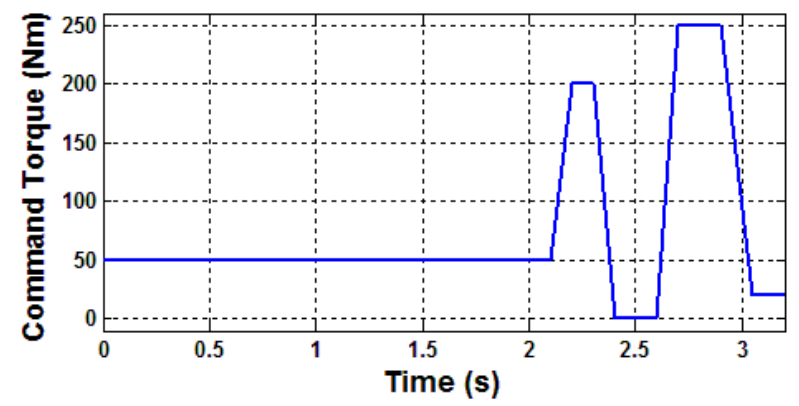

Fig. 4. Load torque profile.

This paper proposes a compensation method based on filter characteristics, which is much more robust to speed variations and has far better performance than the previous methods. The filter used in the SMO is a second-order low-pass discrete Butterworth filter (FIR design). Using this filter as an example, the proposed compensation algorithm is illustrated as follows.

The transfer function of the second-order low-pass Butterworth filter is:

$$
T f(s)=A \frac{1}{s_{L}^{2}+\sqrt{2} s_{L}+1}=A \frac{1}{s^{2}+\sqrt{2} s \omega_{c}+\omega_{c}^{2}}
$$

where $s_{L}=s / \omega_{c}$; and $\omega_{c}$ is the cutoff frequency of the filter. Replacing $s$ with $j \omega$, then delayed angle can be obtained as:

$$
\Delta \theta=-\tan ^{-1}\left(\frac{\sqrt{2} \omega \omega_{c}}{\omega_{c}^{2}-\omega^{2}}\right)
$$

where $\omega$ is the rotor electrical frequency and $\omega=\omega_{r m} \times p / 60$; $\omega_{r m}$ is the rotor speed in RPM and $p$ is the number of pole pairs.

Equation (10) represents the phase shift frequency characteristics of the second-order low-pass filter. Fig. 5 compares the compensated phase delay from (10) and the position error in Fig. 3. The results show these two curves are on top of each other, except for the small oscillations in the position error curve at low-speed regions. Therefore, (10) can be used to correctly compensate for the position error caused by the delay of the filter. The position error after compensation is shown in Fig. 6, which is less than 2 degrees in medium to high-speed regions.

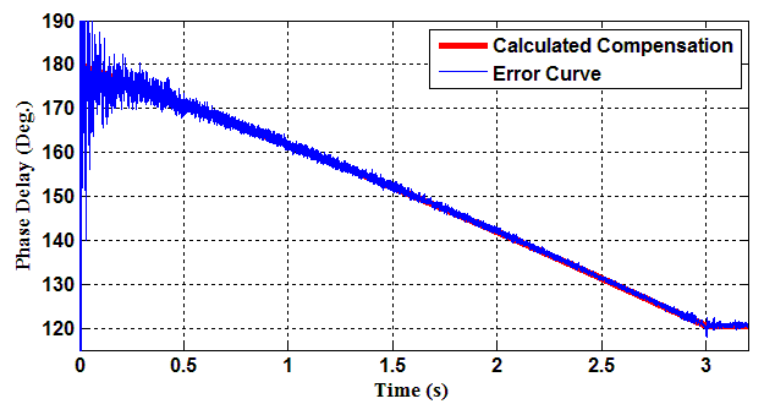

Fig. 5. Comparison between position error and calculated compensation.

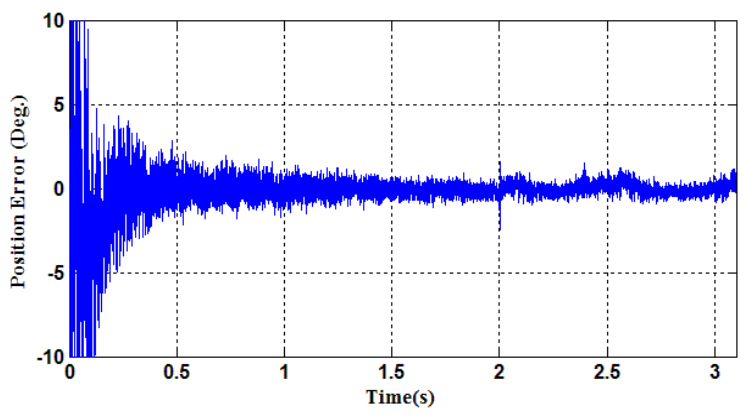

Fig. 6. The position error after compensation.

\section{Multistage Filter}

As shown in Fig. 6, although the phase delay caused by the filter has been well compensated, the performance of the SMO can still be improved. For example, the oscillation of the compensated position error is much larger in the low-speed region than in the high-speed region due to the use of a fixed cutoff frequency for the filter. For example, if the maximum speed is 6,000 RPM, which corresponds to $400 \mathrm{~Hz}$ for a 4 polepair machine, the cutoff frequency of the filter can be set a little higher than $400 \mathrm{~Hz}$, e.g., $450 \mathrm{~Hz}$. However, $450 \mathrm{~Hz}$ is too large for the low-speed region; and a large amount of unwanted harmonic components will pass through the filter, which shall degrade the performance of the SMO in the low-speed region.

TABLE I. SPEED RANGES AND CORRESPONDING CUTOFF FREQUENCIES FOR THE MUlTistaGE FILTER

\begin{tabular}{c|c|c|c|c|c}
\hline $\begin{array}{c}\text { Stage } \\
\text { No. }\end{array}$ & Speed Range & $\begin{array}{c}\text { Boundary } \\
\text { point (RPM) }\end{array}$ & $\begin{array}{c}\text { Hysteresis } \\
\text { band }\end{array}$ & $\begin{array}{c}\text { Elec. } \\
\text { Freq. }\end{array}$ & $\begin{array}{c}\text { Cutoff } \\
\text { Freq. (Hz) }\end{array}$ \\
\hline 1 & $0 \sim 500$ & 0 & 0 & 33.3 & 40 \\
\hline 2 & $500 \sim 900$ & 500 & $450 \sim 550$ & 60 & 80 \\
\hline 3 & $900 \sim 1,600$ & 900 & $850 \sim 950$ & 106.7 & 120 \\
\hline 4 & $1,600 \sim 2,500$ & 1600 & $1,550 \sim 1,650$ & 166.7 & 180 \\
\hline 5 & $2,500 \sim 4,000$ & 2500 & $2,450 \sim 2,550$ & 266.7 & 300 \\
\hline 6 & $>4,000$ & 4000 & $3,950 \sim 4,050$ & N/A & 600 \\
\hline
\end{tabular}

There are many methods to solve this problem. The most straightforward method might be using an adaptive filter, which has a variable cutoff frequency with respect to the rotor 
TABLE II

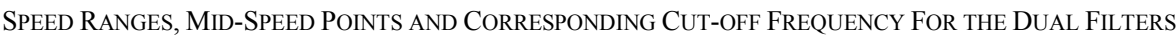

\begin{tabular}{|c|c|c|c|c|c|c|c|c|}
\hline $\mathrm{I}$ & II & III & IV & \multicolumn{2}{|c|}{$\mathrm{V}$} & \multicolumn{2}{|c|}{ VI } & VII \\
\hline & & & & \multicolumn{2}{|c|}{ Cutoff Frequency } & \multicolumn{2}{|c|}{ Cutoff Frequency Combination } & \\
\hline Speed Range & $\begin{array}{c}\text { Stage } \\
\text { Number }\end{array}$ & $\begin{array}{l}\text { Mid-Speed } \\
\text { Points }\end{array}$ & $\begin{array}{c}\text { Modified Speed } \\
\text { Range }\end{array}$ & Filter 1 & Filter 2 & Filter 1 & Filter 2 & $\begin{array}{c}\text { Case } \\
\text { Number }\end{array}$ \\
\hline $0 \sim 500$ & 0 & 0 & $0 \sim 500$ & 40 & 80 & \multirow[b]{2}{*}{40} & \multirow[b]{2}{*}{80} & \multirow[b]{2}{*}{0} \\
\hline \multirow[b]{2}{*}{$500 \sim 900$} & \multirow[b]{2}{*}{1} & \multirow[b]{2}{*}{700} & $500 \sim 700$ & 40 & 80 & & & \\
\hline & & & $700 \sim 900$ & 120 & 80 & \multirow[b]{2}{*}{120} & \multirow[b]{2}{*}{80} & \multirow[b]{2}{*}{1} \\
\hline \multirow[b]{2}{*}{$900 \sim 1,600$} & \multirow[b]{2}{*}{2} & \multirow[b]{2}{*}{1,250} & $900 \sim 1,250$ & 120 & 80 & & & \\
\hline & & & $1,250 \sim 1,600$ & 120 & 180 & \multirow[b]{2}{*}{120} & \multirow[b]{2}{*}{180} & \multirow[b]{2}{*}{2} \\
\hline \multirow[b]{2}{*}{$1,600 \sim 2,500$} & \multirow[b]{2}{*}{3} & \multirow[b]{2}{*}{2,050} & $1,600 \sim 2,050$ & 120 & 180 & & & \\
\hline & & & $2,050 \sim 2,500$ & 300 & 180 & \multirow[b]{2}{*}{300} & \multirow[b]{2}{*}{180} & \multirow[b]{2}{*}{3} \\
\hline \multirow[b]{2}{*}{$2,500 \sim 4,000$} & \multirow[b]{2}{*}{4} & \multirow[b]{2}{*}{3,250} & $2,500 \sim 3,250$ & 300 & 180 & & & \\
\hline & & & $3,250 \sim 4,000$ & 300 & 600 & \multirow[b]{2}{*}{300} & \multirow[b]{2}{*}{600} & \multirow[b]{2}{*}{4} \\
\hline $4,000 \sim 10,000$ & 5 & 10,000 & $4,000 \sim 10,000$ & 300 & 600 & & & \\
\hline
\end{tabular}

speed. However, implementing an adaptive filter will consume more computational source and make the implementation more complex. This paper proposes a multistage filter, where each stage of the filter corresponds to a certain speed region of the IMPSM. Table I lists the stages of the filter used in this paper. The overall range of the rotor speed is divided into six regions. Different cutoff frequencies are used for different speed regions. Although the parameters of the proposed multistage filter will not change as continuously as the adaptive filter, the performance is much better than the filter with only a single constant cutoff frequency.

\section{Dual Filter Structure}

Although the performance of the multistage filter is much better than the filter with a constant cutoff frequency, a transient problem is detected when the filter stage changes abruptly. The transient problem is shown in Fig. 7, in which there is a phase mismatch between the position error and the calculated compensation. A large position error spike occurs at around $0.36 \mathrm{~s}$.

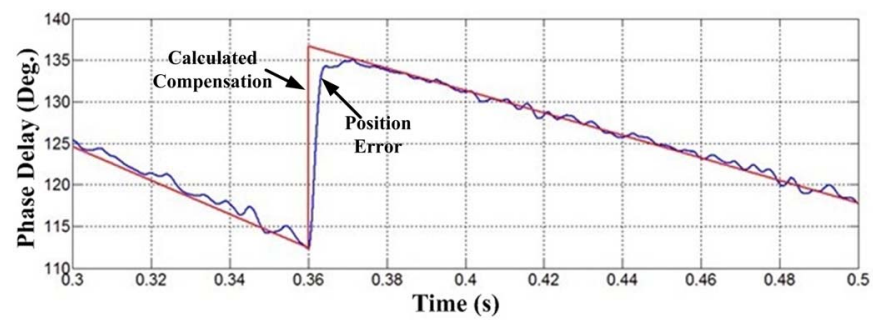

Fig. 7. Transient problem of the multistage filter.

This transient problem can be explained from the point of view of the filter structure. The transfer function of the secondorder filter can be expressed as equation (11), and can also be written as equation (12). If the filter stage changes, the filter parameters will also change. However, the current state $Y[N]$ is calculated by using the previous states $Y[N-1]$ and $Y[N-2]$, which are the states calculated using the filter parameters in previous stages. Due to the transient distortion during the stage transition, the output of the filter cannot directly change from one stage to another. This is the root cause that the filtered position error curve cannot exactly follow the calculated compensation when the filter stage changes.

$$
\begin{gathered}
\frac{Y}{X}=\frac{b_{0}+b_{1} Z^{-1}+b_{2} Z^{-2}}{a_{0}+a_{1} Z^{-1}+a_{2} Z^{-2}} \\
Y[N]=\left(b_{0} X[N]+b_{1} X[N-1]+b_{2} X[N-2]\right. \\
\left.-a_{1} Y[N-1]-a_{2} Y[N-2]\right) / a_{0}
\end{gathered}
$$

To solve this problem, a dual filter structure is proposed. The basic idea is that, if one filter is working with a cutoff frequency for the current speed stage and another filter works in parallel with a cutoff frequency corresponding to the next foreseeable stage, then if the stage changes, the output of the dual filter will also change from that of the current working filter to the parallel filter. By adding a filter in parallel to form a dual filter structure, the transient distortion issue can be seamlessly solved.

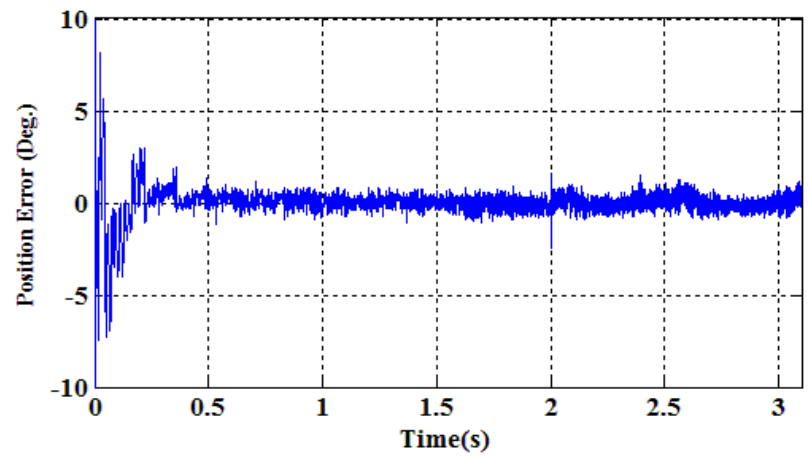

Fig. 8. Compensated position error curve with the multistage dual filters.

The cutoff frequency for each filter and the relationship with respect to the stage number and mid-speed points are listed in Table II. The stage numbers in column II are used to determine the cutoff frequency for the working filter, which is 
shown in column $\mathrm{V}$ and represented by bold red number. This relationship is similar to that shown in Table I. Since the motor speed will increase or decrease, so a mid-speed point is used to determine the cutoff frequency for the filter in the next foreseeable working stage. The mid-speed points are calculated as the mean of the maximum and minimum speeds in each speed range, except for the first stage and last stage. In one speed range, e.g., 1,600 2,500 RPM, if the speed is smaller than 2,050 RPM, which means it is close to 1,600 RPM, and the parallel filter's cutoff frequency is set at $120 \mathrm{~Hz}$; otherwise, the parallel filter's cutoff frequency will be set at $300 \mathrm{~Hz}$. All the cutoff frequencies combinations are shown in column VI, where each combination corresponds to one case shown in column VII. The case number is used to determine the cutoff frequency for the parallel filter. The simulation result is shown in Fig. 8. The large oscillation in the low-speed range and the transient distortion problem are both solved. As shown in Fig. 4 although the load torque changes with a fast slew rate, the performance of the SMO has no degradation.

\section{EXPERIMENT RESULTS}

\section{A. Test setup}

An experimental stand is designed to verify the proposed SMO and the compensation algorithms. In the test stand a prime mover machine and an IPMSM are connected back to back. The prime mover machine is operated in the speedcontrol mode over a wide speed range. The IPMSM works under the torque-control mode. The base torque for the IPMSM is $400 \mathrm{Nm}$ and the base speed is 5,000 RPM. Considering the efficiency, switching noise, and EMI issues, the PWM switching frequency is adaptively adjusted according to the speed from $2 \mathrm{kHz}$ up to $6.5 \mathrm{kHz}$. The sampling frequency of the current and rotor position is the same as the PWM frequency, and the SMO is also executed once per PWM cycle to calculate the estimated rotor position. Other parameters of the IPMSM are the same as the parameters of the simulation model in Section V-A.

\section{B. Experiment Results}

Fig. 9 shows the profiles of the estimated back EMF $e_{\alpha}$ and $e_{\beta}$, as well as the estimated and measured positions, when the IPMSM is operated around 1,000RPM. In this case the cutoff frequency is selected as a constant of $300 \mathrm{~Hz}$, which is much larger than the fundamental frequency of the back EMF. It is obvious to see that the estimated back EMF has larger distortions, which will bring large noise to the estimated position. The position error is large and has obvious oscillations.

As a comparison, if the multistage dual filters are used, Figs. 10 and 11 show the open-loop experiment results at 1,000RPM and 4,800RPM, respectively. Phase delays caused by the filter have been well compensated. The sampling rate of the SMO is kept the same at $6 \mathrm{kHz}$, when the rotor speed changes. As shown in Fig. 11, the estimated back EMF becomes discontinuous at 4,800 RPM when using a sampling rate of $6 \mathrm{kHz}$, because there are only 19 sample points in each electrical revolution. However, if well compensated, the SMO in the high-speed range still has comparable performance as in the lower-speed range. The error between the measured and estimated positions is plotted in Fig. 12. The error is limited within \pm 3 electric degrees at 1,000 RPM and within \pm 5 electric degrees at 4,800 RPM.

As previously mentioned, if well compensated, the proposed SMO is robust to load change, which is verified in Fig. 13. In Fig. 13, a $20 \%$ torque (i.e., $80 \mathrm{Nm}$ ) is added. Compared with the free-shaft operation, the error between the estimated and measured positions is slightly affected. The high-frequency noise in the estimated back EMF is caused by the high-frequency noise in the DAC channel of the oscilloscope, which does not exist in the controller.

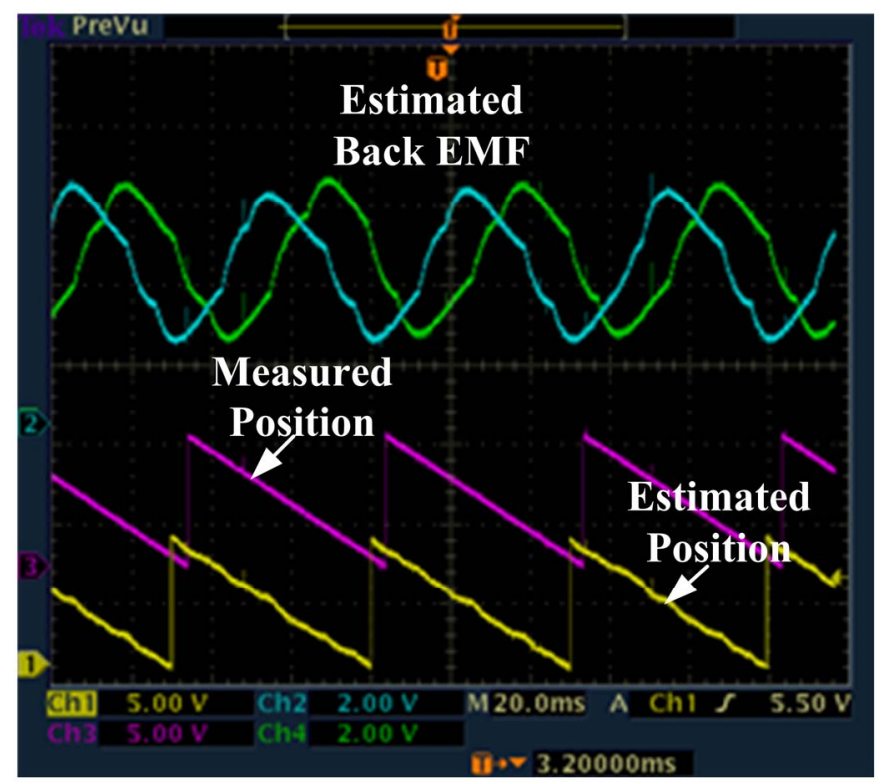

Fig. 9. Estimated back EMF $E_{\alpha \beta}$, estimated rotor position, and measured rotor position from the resolver (1,000 RPM, $0 \mathrm{Nm}$, fixed filter with $\left.\omega_{c}=300 \mathrm{~Hz}\right)$.

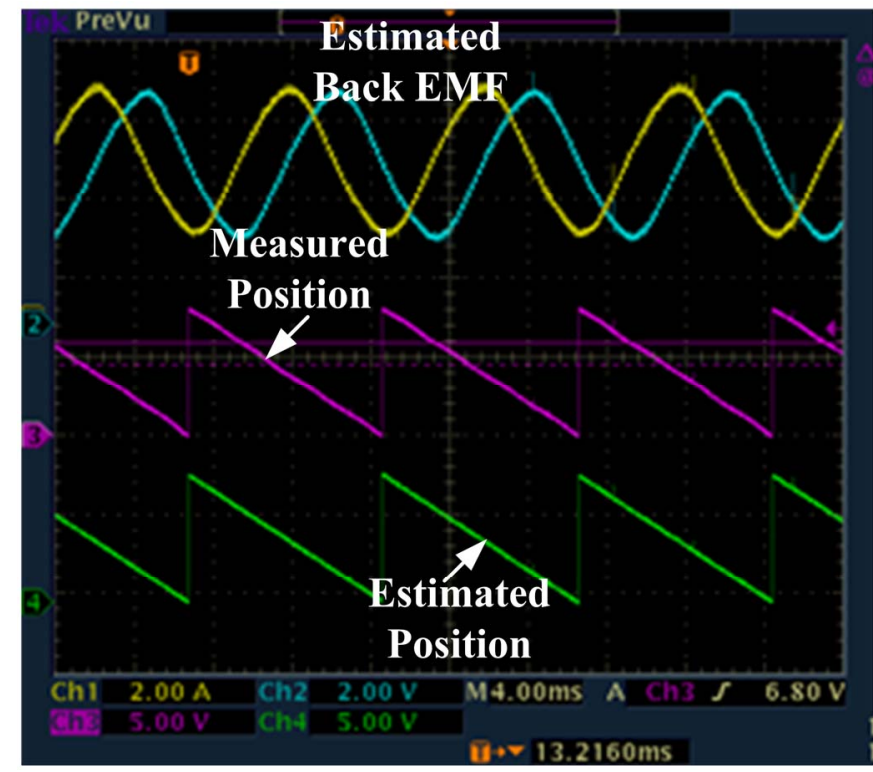

Fig. 10. Estimated back EMF $E_{\alpha \beta}$, estimated rotor position, and measured rotor position from the resolver (1,000 RPM, $0 \mathrm{Nm}$, multistage dual filters). 


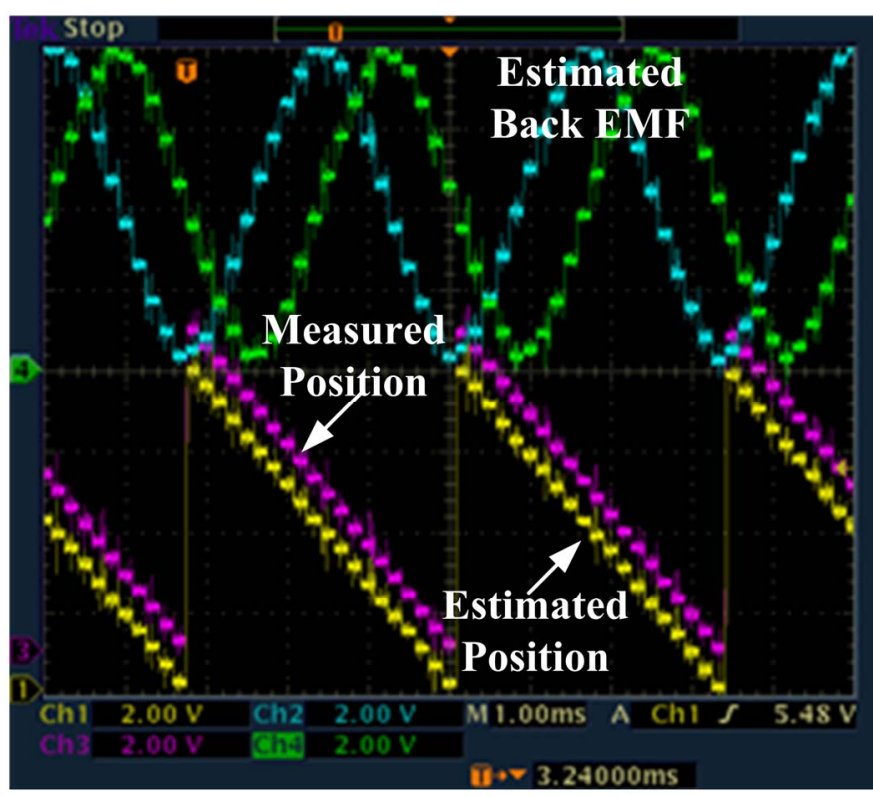

Fig. 11. Estimated back EMF $E_{\alpha \beta}$, estimated rotor position, and measured rotor position from the resolver (4,800 RPM, $0 \mathrm{Nm}$, multistage dual filters).

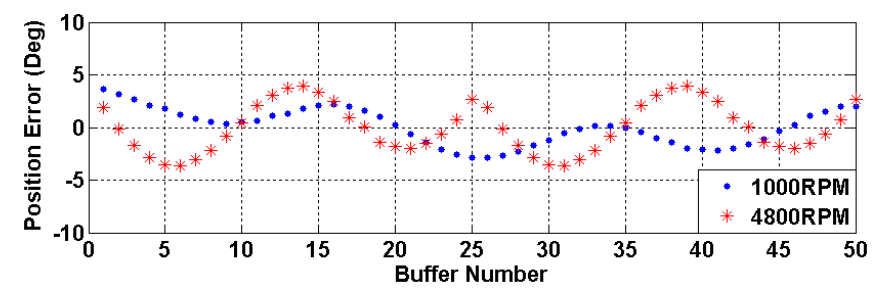

Fig. 12. Error between the estimated and measured positions.

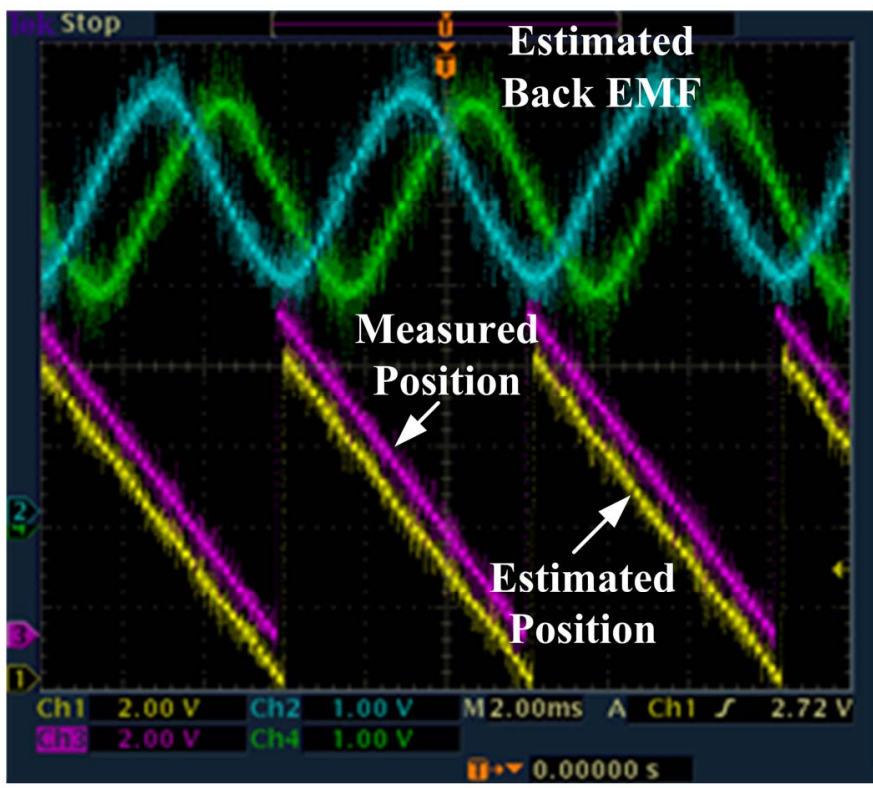

Fig. 13. Estimated back EMF $E_{\alpha \beta}$, estimated rotor position, and measured rotor position from the resolver (1,000 RPM, $80 \mathrm{Nm}$, multistage dual filters).

\section{CONCLUSION}

An improved sliding mode rotor position observer for sensorless control of IPMSMs has been proposed in this paper. In order to enhance the performance of the proposed SMO, a new phase compensation method based on filter characteristics has been proposed. Multistage dual filters have been designed further improve the transient and speed-adaption performance of the phase compensated SMO. The SMO with the new multistage dual filter-based compensation algorithm has been verified by both simulation and experiment results. The improved SMO structure has consistently good rotor position estimation performance over a wide speed range, and is also robust to load changes.

\section{ACKNOWLEDGEMENT}

The authors gratefully acknowledge financial support for this work from Phoenix International - A John Deere Company.

\section{REFERENCES}

[1] M. Pacas, "Sensorless drives in industrial applications," IEEE Industrial Electronics Magazine, vol. 5, no.2, pp. 16-23, June 2011.

[2] Z. Chen, M. Tomita, S. Doki, and S. Okuma, "An extended electromotive force model for sensorless control of interior permanentmagnet synchronous motors," IEEE Trans. Industrial Electronics, vol. 50, no. 20, pp. 288-295, Apr 2003.

[3] S. Chi, Z. Zhang, and L. Xu, "Sliding-mode sensorless control of directdrive PM synchronous motors for washing machine Applications," IEEE Trans. Industry Applications, vol. 45, no. 2, pp. 582-590, Mar-Apr 2009.

[4] S. Morimoto, K. Kawamoto, M. Sanada, and Y. Takeda, "Sensorless control strategy for salient-pole PMSM based on extended EMF in rotating reference frame," in Proc. 2001 IEEE IAS Annual Meeting, vol. 4, pp. 2637-2644, 2011.

[5] M.J. Corley and R.D. Lorenz, "Rotor position and velocity estimation for a salient-pole permanent magnet synchronous machine at standstill and high speeds," IEEE Trans. Industry Applications, vol. 34, no. 4, pp.784-789, Jul/Aug 1998 .

[6] S. Shinnaka, "New 'D-state-observer' based sensorless vector control for permanent magnet synchronous motors," in Proc. 2004 IEEE IAS Annual Meeting, vol. 2, pp. 1000- 1007, 2004.

[7] Y. Zhang and V. Utkin, "Sliding mode observers for electric machinesan overview," in Proc. 2002 IEEE IAS Annual Meeting, pp. 1842-1847, 2004.

[8] S. Chi and L. Xu, "Position sensorless control of PMSM based on a novel sliding mode observer over wide speed range," in Proc. 2006 Power Electronics and Motion Control Conference, vol. 3, pp. 1-7.

[9] M. Elbuluk and C. Li, "Sliding mode observer for wide-speed sensorless control of PMSM drives," In Proc. 2003 IAS Annual Meeting, vol. 1, pp. 480- 485, 2003.

[10] G. Foo and M. F. Rahman, "Sensorless sliding-mode MTPA control of an IPM synchronous motor drive using a sliding-mode observer and HF signal injection," IEEE Trans. Industrial Electronics, vol. 57, no. 4, pp. 1270-1278, Apr 2010.

[11] V. Utkin, J. Guldner, and J. Shi, Sliding Mode Control in Electromechanical Systems, 1st ed. New York: Taylor \& Francis, 1999.

[12] B. Cheng and T.R. Tesch, "Torque feedforward control technique for permanent-magnet synchronous motors," IEEE Trans. Industrial Electronics, vol. 57, pp. 969-974, Mar 2010.

[13] W. Qiao, X. Yang, and X. Gong, "Wind speed and rotor position sensorless control for direct-drive PMG wind turbines," IEEE Trans. Industry Applications, vol. 48, no. 1, pp. 3-11, Jan./Feb. 2012. 